\title{
Yahoo! Digits: A Design Driven to Provide Instant 'Data Driven Insights' and its Use in User Experience Design
}

\author{
Abhishek Kumar \\ Yahoo! R \& D \\ Torrey Pines, EGL, Ring Road, Bangalore \\ abhik@yahoo-inc.com
}

\begin{abstract}
Yahoo! Digits is an internal web analytics tools designed to provide data driven insights for Yahoo! products. The paper outlines the principles of Digits design involving - personalization, insight driven information design and scalable $\mathrm{UI}$ architecture. It also focuses on how Digits is helping designers in understanding their product and users better. Data analysis can be used for evaluating design impact and drives design insights; along with other qualitative methods like user testing, user research, ethnography etc. Discussing data driven design, paper primarily outlines the two essential methods of using data - one as a support to a known issue where data is used to prove and/or understand the scale of a known issue. Other is to drive insights through analysis of data, which the paper terms as 'data driven insight'. The data can be used in understanding user demographics, engagement, customer insights, comparative benchmarking, etc. The paper talks about how the core analytics principles of metric, dimension and friends metric can be used for data driven insights. It also highlights how Digits provides 'context driven' information design and features to help provide data driven insights instantly.
\end{abstract}

Data Driven Design, Analytics, User Experience, Data Presentation

\section{INTRODUCTION}

Yahoo! is an internet company providing solutions for communication, search, entertainment etc. For a company like Yahoo! it becomes essential to track the performance of their products. Yahoo! Digits is the next generation web analytic application built completely out of Bangalore office.

This paper will discuss various aspects of Digits design and also will focus on how digits is helping designers understand their product and helping in design decisions. Paper discusses analytics in a generic fashion so that design community outside Yahoo! can also use the principles of analytics through other analytics tool available in the market.

\section{THE PROCESS \& USER PROFILES}

Digits used a user centred design process; which involved early research on the existing tools pattern of usage through data analysis of the existing older tools and user interviews, followed by product conceptualization, use cases detailing, product flows, wireframes, visual design \& usability testing, followed by improvements. The most important part of the redesign was to focus on the 'users' - their understanding of data and usage patterns.

During the initial user research on data analytics the team realized that there are a large majority of employees in Yahoo! who are not trained in data analysis. To add to the problem if the analytics application itself become very complex and difficult to use there will be a good majority of users who will not be able to use the data.

Looking at the pattern of usage and the profile of the users - which involved Business Unit heads, product managers, product teams (engineers/designers), content writers, analyst, sales and marketing - Digits team identified that most of them would be interested in looking at a specific set of products and data.

In general it was found that designers are not trained into web analytics thus it's important that the application be simple to use. Thus, the most critical aspect of the design was to make the application very easy to use, so that users spend more time in analysing the data rather than to struggle with the application itself. 


\section{DESIGN CONCEPT}

\subsection{Personalization}

Realizing that most user are untrained in analytics, the team decided to build the application that remembers the usage and helps these users reach to the reports easily. The whole design is built that helps users quickly reach out to the relevant reports and spend more time analyzing the data. There are 6 different levels of personalization bookmarking, share reports, recent reports, recently viewed pages/products, auto redirect to last seen report. Another important aspect was transfer of settings, e.g. sales person is looking at Page Views report for Mail for an age segment of 12-24, which comes from India. Now the user moves to another product like News the same settings of age segment and country is transferred to News data.

\subsection{Insight Driven Design}

\subsubsection{Kind of Reports}

\subsubsection{Summary Reports}

Yahoo! before Digits had an older generation of analytics. Looking at the data available on the older analytics application it became evident that usage of summary reporting is at least 10 times more than any other section. Summary Report provides instant insights to product and page reporting.

Summary Report in Digits has primarily two sections - the top one shows all the metrics available for a product/page. This section is called All Metric Snapshot.

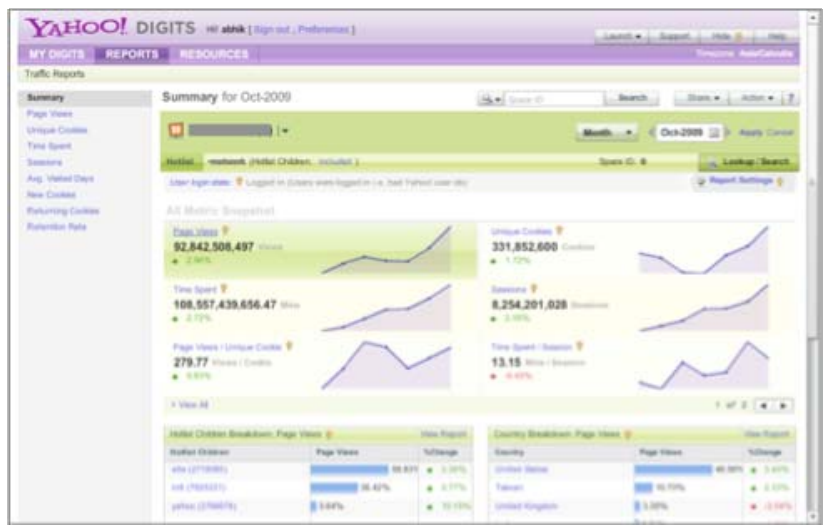

Figure 1: Screenshot of Summary Report

The bottom part contain modules that essentially answers some basic questions like "Who is coming to my site" "where are the users coming from" and "what part of my site are more popular". These modules are driven from the metric selected on the top section - All Metric Snapshot (Fig 1).

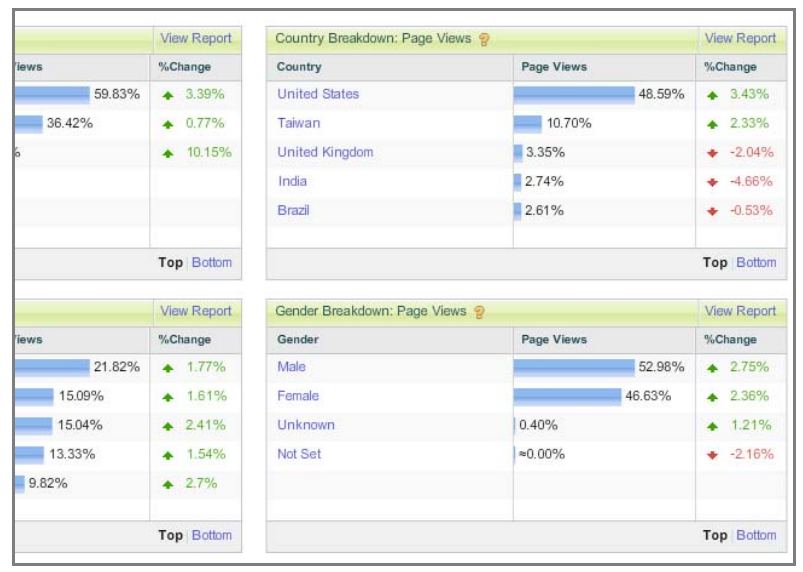

Figure 2: Modules on the Summary Page

Summary Report is helping the product teams and designers to quickly understand the demographics of users on their products, popular sections of the site and the region (country) of traffic (Fig2).

The real value is provided through the interactivity of the page, which allows to see the modules for any shown metric. E.g. designers can now easily discover content and demographics, which provide more Page Views versus demographics and content where users spend more Time.

This insight can be used in choosing what sections of the page should have more engagement while what potions could be used to increase Page Views.

\subsubsection{2. (Metric) Detail Page}

As the Summary Report is designed to give an overall view of the performance of the product/pages, Detail Page is designed to drill down into the metric data.

Information on the Detail Page contains two sections. The top section provides the total count, e.g. in the case of Page Views, total value is mentioned. To provide some context the metric count is accompanied by other metrics (called Friend Metric).

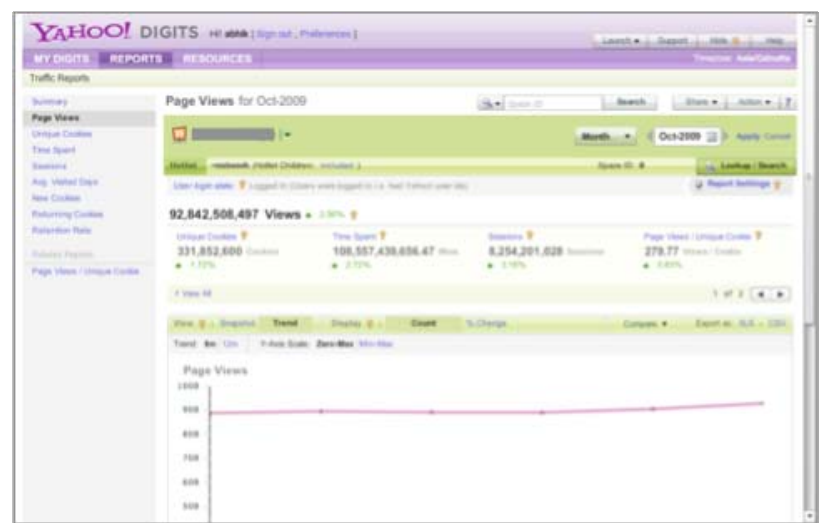

Figure 3: Screenshot of Detail Page 
The bottom portion is totally dedicated to the details of the metric; which shows the trending graph, data table and other actions required to analyze the data (Fig 3).

Along with normal mode, Detail Page also allows advanced segmentation through Breakdowns and Filters. Where breakdown essentially breaks down the metric with chosen dimensions like Age, Gender, Country and Content. Filter allows the user to filter the metric with the above dimensions (Fig 4). These 'advanced settings' by default are hidden from the normal users so that they don't get overwhelmed with the settings option. These are required for advanced reporting, thus their usage is limited to advanced users.

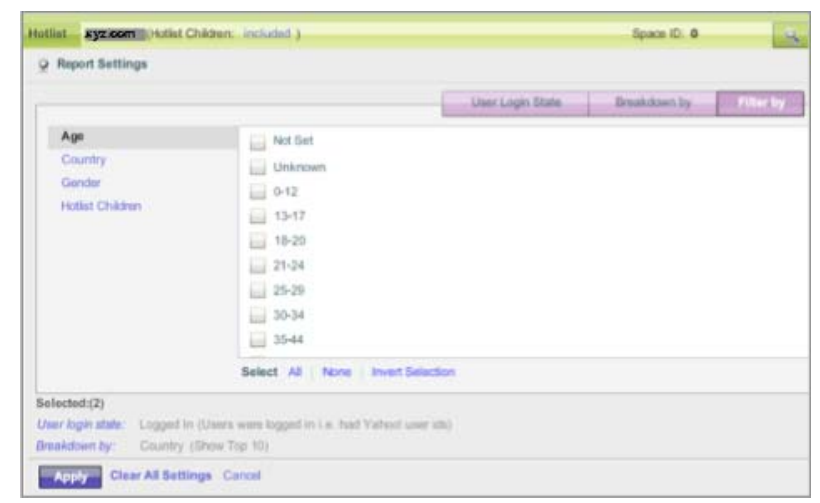

Figure 4: Breakdown and Filter settings in Detail Report.

E.g. Page Views (PVs) Breakdown by Age - will be Page Views contribution of various age segments for Mail users. This report will split the total Page View (PVs) with contribution of all the age segments (Fig 5).

\begin{tabular}{|l|l|}
\hline V Table B & \\
\hline Age & Page Views for Oct-2009 (\%) \\
\hline $35-44$ & $21.82 \%$ \\
$25-29$ & $15.09 \%$ \\
$45-54$ & $15.04 \%$ \\
$30-34$ & $13.33 \%$ \\
$21-24$ & $9.82 \%$ \\
$55-64$ & $8.81 \%$ \\
$18-20$ & $5.73 \%$ \\
\hline
\end{tabular}

Figure 5: Showing the age group contribution for Page Views

Filter PVs by age segment of $18-29$, will show only the PVs driven by that segment.

Detail Page along with its functionality to see the metric in context of other metrics and Compare feature is making it simpler for designers to get instant insights. E.g. now designers can compare this year's trend with previous years trend to see the seasonality in data. Designers can now account for seasonal trends in data and make better predictions on the traffic.

Along with that, due to filtering and breakdown capabilities designers can now see the data and the trends for a specific demographics and content. E.g. designers can track the usage patterns of teen-age audience on the site through Filter. Thus, the designers can understand the behaviour of a user segment and can design for it with better understanding.

\subsubsection{Information hierarchy}

Digits design is built with core principle that the most important information should stand out. Example, for any metric report like Page View the core information that should stand out is the total number of Page Views. Other supporting information is toned down so that the actual numbers stand out. There are three different levels used in Digits:

\subsubsection{Primary data}

Bigger font and bolded generally visible even from a distance (Fig 6).

\begin{tabular}{|lc|}
\hline $\mathbf{9 2 , 8 4 2 , 5 0 8 , 4 9 7}$ Views $+2.56 \%$ & 8 \\
\hline Unique Cookies \& & Time Spent \& \\
$331,852,600$ Cookies & $108,557,439,656$. \\
$41.72 \%$ & $+2.72 \%$ \\
\hline
\end{tabular}

Figure 6: Primary and Secondary data, Black and bolded data is the primary data while \%age change and other metric constitutes the secondary data.

\subsubsection{Secondary data:}

Generally supporting information like \% change or other supporting metric data (Fig 6).

\subsubsection{Roll over data}

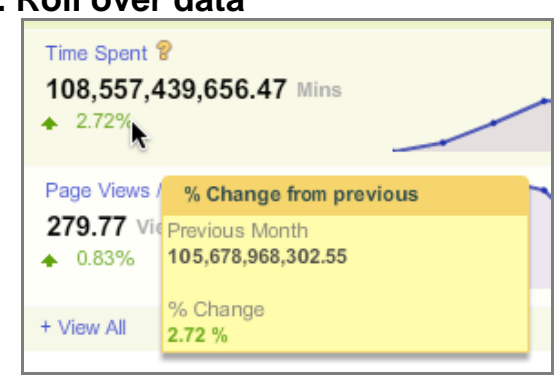

Figure 7: Roll over percentage change data in All Metric Snapshot section of Summary Report 


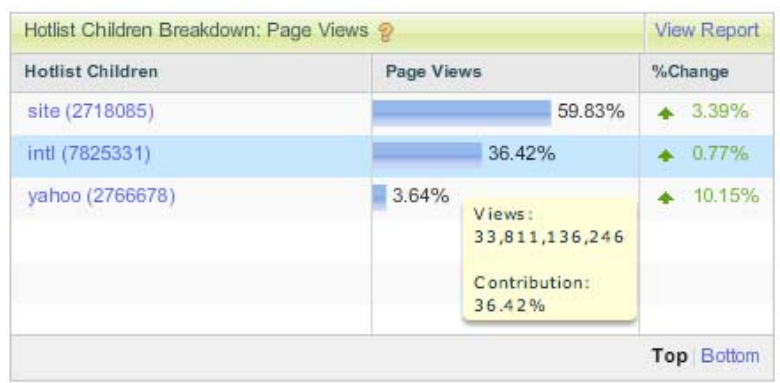

Figure 8: Roll over data on Modules in the Summary Report, the actual data shown on the $\mathrm{UI}$ is in percent contribution while the actual count is shown on roll over

This data is generally not visible on the UI upfront but shown on user action - mouse over the numbers (Fig $7 \& 8$ ).

During early research it was discovered that showing too much information scares the users who are not trained in analytics. This hierarchy is helping designers focus on the most critical information and reducing the information overload.

\subsubsection{Context}

Both in the summary reports where multiple metrics are shown are always accompanied by \%age change and some historical trend. E.g. 10,000 PVs will not make sense unless you know more information about it. Like whether it's gone up or down (\%age change), along with some sense of historical data (spark line showing 7 historical data points) (Fig 9).

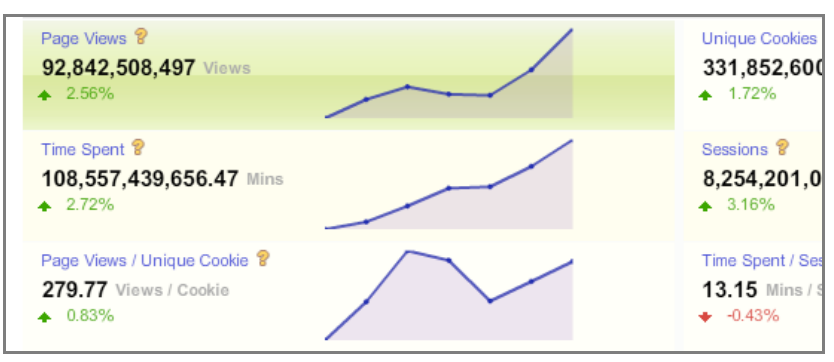

Figure 9: All Metric Snapshot

Context is the most important part of analytics. Without considering the context both of factors within the product and outside (competitors or events) it become very difficult to make a correct judgment on the data.

A simpler data presentation and compare is helping designers to better understand the data.

\subsubsection{Friend Metric}

Along with the chosen metric, Digits allows the ability to see another metric to help setup the context. Friend Metric are shown on Summary section of the Detail Page and also in the Data table (Fig 10 \& 11). Like, PVs can be seen along with Time Spent. The use case could be that the
PVs are increasing while the Time Spent is decreasing, which might indicate that engagement in the site might be going down. Or the site is not getting the desired traffic. User are coming to the site and not finding relevant information/content to keep them engaged on the site.

\begin{tabular}{|llr|}
\hline $\mathbf{9 2 , 8 4 2 , 5 0 8 , 4 9 7}$ Views $* 2.56 \%$ & & \\
\hline Unique Cookies \& & Time Spent \& & Session \\
$331,852,600$ Cookies & $108,557,439,656.47$ Mins & 8,254 \\
$41.72 \%$ & $* 2.72 \%$ & $* 3.16$ \\
\hline
\end{tabular}

Figure 10: Summary section in the Detail Page showing Friend metric along side Page Views count.

This ability to see two metric at a time allows for the discovery of insights like, $20 \%$ users (say 20-25 age group) is giving $70 \%$ PVs. This will be an important insight, as now the designer knows that if this $20 \%$ user group leaves the site, the PVs will drop by $70 \%$.

\begin{tabular}{|c|c|c|}
\hline Page Views for Oct-2009 (\%) & V Time Spent & - \\
\hline $48.59 \%$ & 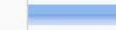 & \\
\hline $10.70 \%$ & $=7.27 \%$ & \\
\hline $3.35 \%$ & $4.00 \%$ & \\
\hline $2.74 \%$ & $2.24 \%$ & \\
\hline $2.61 \%$ & $2.46 \%$ & \\
\hline
\end{tabular}

Figure 11: Data table with Friend Metric. The last column has a drop down with all the Friend Metrics.

\subsubsection{Percentage contribution vs. actual count}

Digits use a good balance between \%age contributions vs. actual count. In Summary Report, Digits use \%age contribution to understand the split. There are cases where \%age contribution is more useful than actual numbers. Information design is built around this understanding of where to use \%age as default vs. actual count. E.g. if we say $12-25$ age group is giving $12,234 \mathrm{PVs}$, it would be difficult to interpret. While if we say $12-25$ age group contributes $45 \%$ of the overall PVs, it might be more actionable insight.

\subsection{Scalability}

Another important aspect of Digits design was its capability to scale. Most often we see the design not able to accommodate the requirements and thus becomes a potential case for a redesign. Frequent redesigning can take up crucial time and hold you back for a period where your competitors can forge ahead with more functionality. All the components used in the design can allow to scale. The UI uses scalable Tab navigation and carousal to help the UI scale in future. 


\section{USING DATA IN DESIGN}

Design evaluation and research uses mostly qualitative methods like ethnographic research, usability testing, user interviews, contextual inquiry etc.

Data can be used in two essential ways - one to support an existing issue found through qualitative methods. For example at Yahoo! two of the designers found an issue with password recovery tool through user research. They found that most users are not able to successfully recover the password. They informed the team working on it, but could not convince the team to take this up as high priority. They then looked at the flow analysis and used the data to convince the team about the scale of the problem. The issue was reprioritized to the highest and quickly fixed.

Another way to look for insight is through the data itself. For example in one of the news site the team found that the traffic peak around 9:00am while the site was uploading the fresh content around 11:00am. Realizing that most users are not getting the fresh content the team preponed the content release.

Data analysis can be used either to support design issue or to identify design issues.

\section{WHAT DESIGNERS NEED TO KNOW}

\subsection{Definition of Metric}

It's important for designers to understand the definition of the metric. To be able to make sense of the number it's important to know how it's calculated. For examples its useful to know what does Time Spent mean and how its calculated to make sense of the numbers.

On mouse over on metric title Digits shows up the definition of that metric (Fig 12).

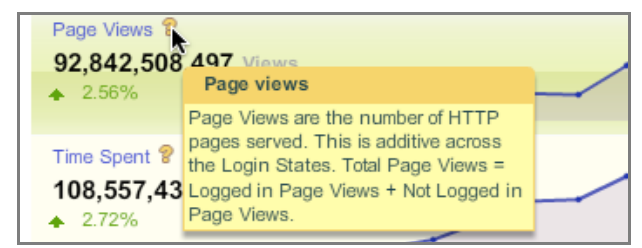

Figure 12: Metric definition

\subsection{Context creates actionable insights}

Context is important because it's difficult to make sense of the number without it. Assume the report says Monthly Page Views 2,233,233; with just this piece of information its not very easy to make any insight. Now if the data say $2,233,233(+10 \%)$ then it brings some content. So as we start to add more data to the number like 10 historical data points, PVs of competitors, last year's data or PVs along with other metric the data becomes more actionable.

This is the whole premise of Digits design, to always provide number along with features that allows set this context - through either friend metric or compare feature.

\subsection{Data can tell you "What" but not "Why"}

It's important to note that data can answer "What" but can not answer "Why" [1]. E.g. Data can tell you that PVs are going down but "Why" it's going down cannot be easily answered by data alone. "Why" can only be answered by either usability testing or other qualitative methods.

\section{CONCLUSION}

Data provides an unbiased view to the performance of the site/application.

Data helps identify gaps and opportunities. It surfaces popular and underperforming parts of the site/application.

Data provides a good understanding of user segments and demographics.

Data can help drive Design Strategies - designers can choose to leverage the top user group or to cover up other underperforming user groups. Example, a site might want to target the user group that's giving the best revenue and make the site more relevant and engaging to that user segment. Or can decide to broaden the user segment and try to find gaps to make it more relevant to broaden the user segment.

Data can support user research outcomes and bring more authenticity to usability findings.

In order to make the usage of data more widely used, web analytics application need to be designed in way that are simpler to use and shows data is a way that makes it easier to interpret.

Design community should start to look into data and some basic analytics should be thought in design schools to make the next generation of designers more comfortable in data analysis.

Digits usage doubled compare to the older application in just two months of the launch. User feedback has been very positive across profiles with special mention on ease of use, sleek look $n$ feel and the effective data presentation on the UI. 


\section{ACKNOWLEDGEMENTS}

Digits would not have been possible without the leadership and support of Girish Krishnan (Product Manager), the whole product team (Engineering and QA) and the product management team in U.S. Special thanks to Sandeep Datar and other UED members for guidance and support.

\section{REFERENCES}

[1] Kaushik, Avinash. Occam's Razor by Avinash Kaushik. http://www.kaushik.net/avinash/

[2] Web Analytics Definition 2008. http://www.webanalyticsassociation.org/attachment s/committees/5/WAA-Standards-AnalyticsDefinitions-Volume-I-20070816.pdf 